\title{
Charge Asymmetries in Semileptonic B Decays
}

\author{
lain Bertram ${ }^{* \dagger}$ \\ Lancaster University \\ E-mail: i.bertramelancaster.ac.uk
}

I present measurements made by the D0 collaboration of the time-integrated flavor-specific semileptonic charge asymmetry in the decays of the $B_{s}^{0}$ and $B_{d}^{0}$ mesons that have undergone flavor mixing, $a_{\mathrm{sl}}^{s}$ and $a_{\mathrm{sl}}^{d}$, using $10.4 \mathrm{fb}^{-1}$ of proton-antiproton collisions collected by the D0 detector during Run II at the Fermilab Tevatron Collider. The results are $a_{\mathrm{sl}}^{s}=$ $[-1.12 \pm 0.74$ (stat) \pm 0.17 (syst) $] \%$ andf $a_{\mathrm{sl}}^{d}=[0.68 \pm 0.45$ (stat.) \pm 0.14 (syst.) $] \%$ which are the two most precise published results and are in agreement with standard model predictions. These results are combined with the like-sign dimuon charge asymmetry, $A_{\mathrm{sl}}^{b}$, and recent preliminary results from the $\mathrm{LHCb}$ and $\mathrm{BaBar}$ experiments to obtain new world averages.

14th International Conference on B-Physics at Hadron Machines April 8-12, 2013

Bologna, Italy

\footnotetext{
*Speaker.

${ }^{\dagger}$ representing the D0 Collaboration.
} 


\section{Introduction}

$\mathrm{CP}$ violation has been observed in the decay and mixing of neutral mesons containing strange, charm and bottom quarks. Currently all measurements of $\mathrm{CP}$ violation, either in decay, mixing or in the interference between the two, have been consistent with the presence of a single phase in the CKM matrix. An observation of anomalously large CP violation in $B_{s}^{0}$ oscillations can indicate the existence of physics beyond the standard model (SM) [1]. Measurements of the like-sign dimuon asymmetry by the D0 Collaboration $[2,3]$ show evidence of anomalously large CP-violating effects using data corresponding to $9 \mathrm{fb}^{-1}$ of integrated luminosity. Assuming that this asymmetry originates from mixed neutral $B$ mesons, the measured value is $A_{\mathrm{sl}}^{b}=C_{d} a_{\mathrm{sl}}^{d}+C_{s} a_{\mathrm{sl}}^{s}=[-0.787 \pm 0.172$ (stat.) \pm 0.021 (syst.) $] \%$, where $a_{s l}^{s(d)}$ is the time-integrated flavor-specific semileptonic charge asymmetry in $B_{s}^{0}\left(B_{d}^{0}\right)$ decays that have undergone flavor mixing and $C_{d(s)}$ is the fraction of $B_{d}^{0}\left(B_{s}^{0}\right)$ events. The value of $a_{\mathrm{sl}}^{s}$ and $a_{\mathrm{sl}}^{d}$ are extracted from this measurement and found to be $a_{\mathrm{sl}}^{s}=(-1.81 \pm 1.06) \%$ and $a_{\mathrm{sl}}^{d}=(-0.12 \pm 0.52) \%$ [3]. This Note presents a short summary of the measurements of $a_{\mathrm{sl}}^{s}$ [4] and $a_{\mathrm{sl}}^{d}$ [5] using the full Tevatron data sample with an integrated luminosity of $10.4 \mathrm{fb}^{-1}$.

The asymmetry $a_{\mathrm{sl}}^{q}$ is defined as

$$
a_{\mathrm{sl}}^{q}=\frac{\Gamma\left(\bar{B}_{q}^{0} \rightarrow B_{q}^{0} \rightarrow \ell^{+} v X\right)-\Gamma\left(B_{q}^{0} \rightarrow \bar{B}_{q}^{0} \rightarrow \ell^{-} \bar{v} \bar{X}\right)}{\Gamma\left(\bar{B}_{q}^{0} \rightarrow B_{q}^{0} \rightarrow \ell^{+} v X\right)+\Gamma\left(B_{q}^{0} \rightarrow \bar{B}_{q}^{0} \rightarrow \ell^{-} \bar{v} \bar{X}\right)},
$$

where in these analyses $\ell=\mu$. The flavor of the $B_{q}^{0}$ meson at the time of decay is identified using the charge of the associated muon, and these analyses do not make use of initial-state tagging. We assume there is no production asymmetry between $B_{q}^{0}$ and $\bar{B}_{q}^{0}$ mesons, that there is no direct CP violation in the decay of charm mesons to the indicated states or in the semileptonic decay of $B_{q}^{0}$ mesons, and that any $\mathrm{CP}$ violation in $B_{q}^{0}$ mesons only occurs in mixing. We also assume that any direct $\mathrm{CP}$ violation in the decay of $b$ baryons and charged $B$ mesons is negligible.

\section{The semi-leptonic charge asymmetry in $B_{s}^{0}$ mesons}

The measurement of the semi-leptonic charge asymmetry in $B_{s}^{0}$ mesons is measured using the decay $B_{s}^{0}\left(\bar{B}_{s}^{0}\right) \rightarrow D_{s}^{\mp} \mu^{ \pm} X$ decays, with $D_{s}^{\mp} \rightarrow \phi \pi^{\mp}$ and $\phi \rightarrow K^{+} K^{-}$. The measurement is performed using the raw asymmetry

$$
A=\frac{N_{\mu^{+} D_{s}^{-}}-N_{\mu^{-} D_{s}^{+}}}{N_{\mu^{+} D_{s}^{-}}+N_{\mu^{-} D_{s}^{+}}}
$$

where $N_{\mu^{+} D_{s}^{-}}\left(N_{\mu^{-} D_{s}^{+}}\right)$is the number of reconstructed $B_{s}^{0} \rightarrow \mu^{+} D_{s}^{-} X\left(\bar{B}_{s}^{0} \rightarrow \mu^{-} D_{s}^{+} X\right)$ decays. The time-integrated flavor-specific semileptonic charge asymmetry in $B_{s}^{0}$ decays which have undergone flavor mixing, $a_{\mathrm{sl}}^{s}$, is then given by

$$
a_{\mathrm{sl}}^{s} \cdot F_{B_{s}^{0}}^{\mathrm{osc}}=A-A_{\mu}-A_{\text {track }}-A_{K K}
$$

where $A_{\mu}$ is the reconstruction asymmetry between positive and negatively charged muons in the detector [6], $A_{\text {track }}$ is the asymmetry between positive and negative tracks, $A_{K K}$ is the residual kaon asymmetry from the decay of the $\phi$ meson, and $F_{B_{s}^{0}}^{\text {osc }}$ is the fraction of $D_{s}^{-} \rightarrow \phi \pi^{-}$decays 
that originate from the decay of a $B_{s}^{0}$ meson after a $\bar{B}_{s}^{0} \rightarrow B_{s}^{0}$ oscillation. The $F_{B_{s}^{0}}^{\text {osc }}$ factor corrects the measured asymmetry for the fraction of events in which the $B_{s}^{0}$ meson is mixed under the assumptions outlined earlier that no other physics asymmetries are present in the other $b$-hadron backgrounds. The fraction of mixed events integrated over time is extracted using Monte Carlo (MC) simulations.

The raw asymmetry (Eq. 2.1) is extracted by fitting the $M\left(\phi \pi^{\mp}\right)$ distribution of the $D_{s}^{\mp}$ candidates using a $\chi^{2}$ minimization. The fit is performed simultaneously, using the same models, on the sum (Fig. 1) and the difference (Fig. 2) of the $M\left(\phi \pi^{-}\right)$distribution associated with a positively charged muon and $M\left(\phi \pi^{+}\right)$distribution associated with a negatively charged muon.

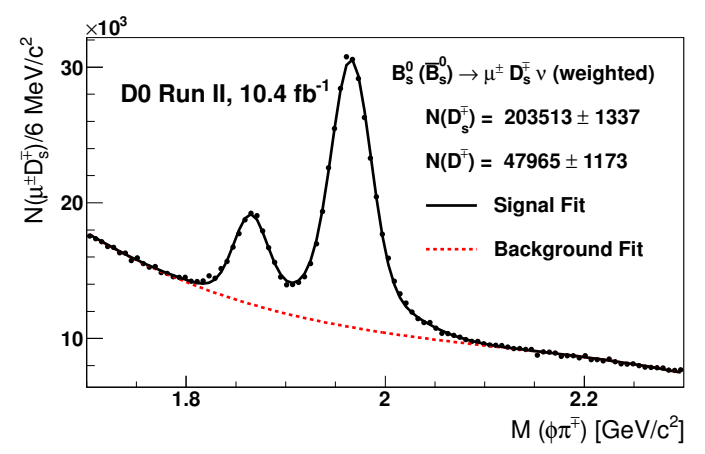

Figure 1: The weighted $K^{+} K^{-} \pi^{\mp}$ invariant mass distribution for the $\mu^{ \pm} \phi \pi^{\mp}$ sample with the solid line representing the signal fit and the dashed line showing the background fit. The lower mass peak is due to the decay $D^{\mp} \rightarrow \phi \pi^{\mp}$ and the second peak is due to the $D_{s}^{\mp}$ meson decay. Note the zerosuppression on the vertical axis.

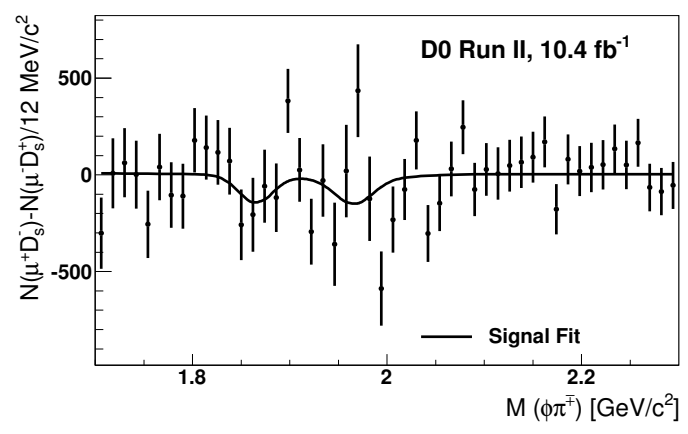

Figure 2: The fit to the difference distribution for the data with the solid line representing the fit (for clarity the data has been rebinned).

The raw asymmetry is corrected by $A_{\mu}+A_{\text {track }}+A_{K K}=[0.13 \pm 0.06$ (syst) $] \%$, including the statistical uncertainties combined in quadrature. The fraction of $B_{s}^{0}$ decays in the sample and the time-integrated oscillation probability, we find $F_{B_{s}^{0}}^{\text {osc }}=0.465$. The resulting time-integrated flavorspecific semileptonic charge asymmetry is found to be

$$
a_{\mathrm{sl}}^{s}=[-1.12 \pm 0.74(\text { stat }) \pm 0.17 \text { (syst) }] \% .
$$

\section{The semi-leptonic charge asymmetry in $B_{d}^{0}$ mesons}

The measurement of the semi-leptonic charge asymmetry in $B_{d}^{0}$ mesons is measured using two separate orthogonal decay channels:

1. $B_{d}^{0} \rightarrow \mu^{+} v D^{-} X$, with $D^{-} \rightarrow K^{+} \pi^{-} \pi^{-}$(plus charge conjugate process);

2. $B_{d}^{0} \rightarrow \mu^{+} v D^{*-} X$, with $D^{*-} \rightarrow \bar{D}^{0} \pi^{-}, \bar{D}^{0} \rightarrow K^{+} \pi^{-}$(plus charge conjugate process);

The two channels are treated separately, with each being used to extract $a_{\mathrm{sl}}^{d}$, before the final measurements are combined. 
The time-integrated flavor-specific semileptonic charge asymmetry in $B_{d}^{0}$ decays which have undergone flavor mixing, $a_{\mathrm{sl}}^{d}$, is then given by

$$
a_{\mathrm{sl}}^{d} \cdot F_{B_{d}^{0}}^{\mathrm{osc}}=A-A_{\mu}-A_{K}+2 A_{\pi}
$$

where $A_{\pi}=A_{\text {track }}$ and $A_{K}$ is the residual kaon asymmetry.

The $B_{d}^{0}$ meson has a mixing frequency $\Delta M_{d}=0.507 \pm 0.004 \mathrm{ps}^{-1}$, of comparable scale to the lifetime $\tau\left(B_{d}^{0}\right)=1.518 \pm 0.007$ ps [9]. Hence the fraction of oscillated $B_{d}^{0}$ mesons is a strong function of the measured decay time. The best precision is obtained by binning the data as a function of the transverse decay length. Small values of the transverse decay length should have negligible contributions from oscillated $B_{d}^{0}$ mesons, and are not included in the final $a_{\mathrm{sl}}^{d}$ measurement. They represent a control region in which the measured raw asymmetry should be dominated by the background contribution.

The raw asymmetries for each decay channel and each decay length bin are extracted using $\chi^{2}$ minimization. The fits are performed simultaneously on the sum and the difference (Fig. 3) of the $\mu D$ and $\mu D^{*}$ distributions.

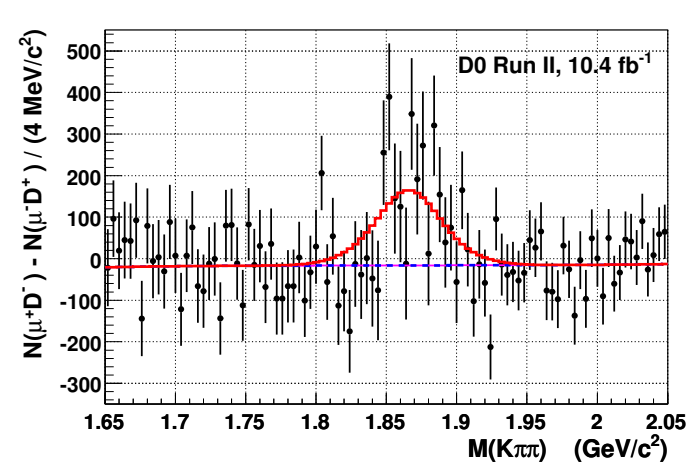

(a) $\mu D$ channel

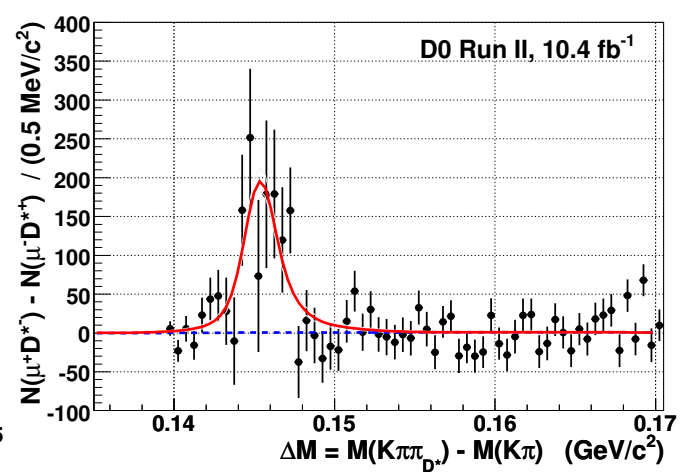

(b) $\mu D^{*}$ channel

Figure 3: Examples of the raw asymmetry fit for the two decays channels, for the fifth visible proper decay length $\left(B_{d}^{0}\right)$ bin corresponding to $\left(0.10<\operatorname{VPDL}\left(B_{d}^{0}\right)<0.20\right) \mathrm{cm}$. The plots show the difference distributions. In both cases, the solid line represents the total fit function, with the background part shown separately by the dashed line.

The extracted raw asymmetries are corrected for background asymmetries on a bin-by-bin basis along with the systematic uncertainties. Once the uncertainties on the individual $a_{\mathrm{sl}}^{d}$ measurements are established, the combination between VPDL bins, and then between channels, is performed. For each channel, the combined $a_{\mathrm{sl}}^{d}$ value is obtained by a weighted average of the four individual measurements where the weights are the inverse of the sum in quadrature of statistical and systematic uncertainties for that measurement. The central values and uncertainties for the combinations are again determined by performing ensemble tests, with all inputs varied, and examining the effect on the final values of $a_{\mathrm{sl}}^{d}$ from each channel. This procedure yields the following results:

$$
\begin{aligned}
a_{\mathrm{sl}}^{d}(\mu D) & =[0.43 \pm 0.63 \text { (stat.) } \pm 0.16 \text { (syst.) }] \% \\
a_{\mathrm{sl}}^{d}\left(\mu D^{*}\right) & =[0.92 \pm 0.62 \text { (stat.) } \pm 0.16 \text { (syst.) })] \%
\end{aligned}
$$


Finally, the combination is extended to give the full weighted average of the two channel-specific measurements, with full propagation of uncertainties, to yield the final measurement:

$$
a_{\mathrm{sl}}^{d}=[0.68 \pm 0.45 \text { (stat.) } \pm 0.14 \text { (syst.) }] \% .
$$

\section{Combination of D0 results}

The D0 measurements of $a_{\mathrm{sl}}^{d}, a_{\mathrm{sl}}^{s}$, can be combined with the two-dimensional constraints on $\left(a_{\mathrm{sl}}^{d}, a_{\mathrm{sl}}^{s}\right)$ from the D0 measurement of the dimuon charge asymmetry $A_{\mathrm{sl}}^{b}$ [3]. The full twodimensional fit yields the following values:

$$
\begin{aligned}
& a_{\mathrm{sl}}^{d}(\mathrm{D} 0 \mathrm{comb})=(0.10 \pm 0.30) \%, \\
& a_{\mathrm{sl}}^{s}(\mathrm{D} 0 \text { comb })=(-1.70 \pm 0.56) \%,
\end{aligned}
$$

with a correlation coefficient of -0.50 . The $\chi^{2}$ of this fit is 2.9 , and the standard model $p$-value is 0.0036 , corresponding to a 2.9 standard deviation effect. Figure 4 shows the two-dimensional contours from this combination.

\section{Updated World Averages}

The D0 measurements of $a_{\mathrm{sl}}^{s}$ and $a_{\mathrm{sl}}^{d}$ can be combined with all other measurements to form updated world averages. I use a simple weighted average, assuming that the measurements are fully independent. The D0 [4] and the preliminary LHCb [7] measurements of $a_{\mathrm{sl}}^{s}$ are combined:

$$
a_{\mathrm{sl}}^{s}(\mathrm{WA})=(-0.60 \pm 0.49) \% .
$$

The B-factories average [?], the D0 [5] and the new preliminary BaBar [8] measurement of $a_{\mathrm{sl}}^{d}$ are also combined,

$$
a_{\mathrm{sl}}^{d}(\mathrm{WA})=(0.23 \pm 0.26) \% .
$$

These numbers can be combined with $A_{\mathrm{sl}}^{b}$, we obtain the following values:

$$
\begin{aligned}
& a_{\mathrm{sl}}^{d}(\mathrm{comb})=(-0.03 \pm 0.21) \%, \\
& a_{\mathrm{sl}}^{s}(\mathrm{comb})=(-1.10 \pm 0.41) \%,
\end{aligned}
$$

where the two parameters have a correlation coefficient of -0.31 . The results are shown in Fig. 5, with the two dimensional contours overlaid on the four constraints from the input measurements. The fit returns a $\chi^{2}$ of 3.8 for 2 degrees-of-freedom. The $p$-value of the combination with respect to the SM point is 0.0148 , corresponding to an inconsistency at the 2.4 standard deviation level.

\section{References}

[1] A. Lenz and U. Nierste, arXiv:1102.4274; A. Lenz and U. Nierste, J. High Energy Phys. 06, 072 (2007).

[2] V. M. Abazov et al. (D0 Collaboration), Phys. Rev. D 82, 032001 (2010); V. M. Abazov et al. (D0 Collaboration), Phys. Rev. Lett. 105, 081801 (2010). 


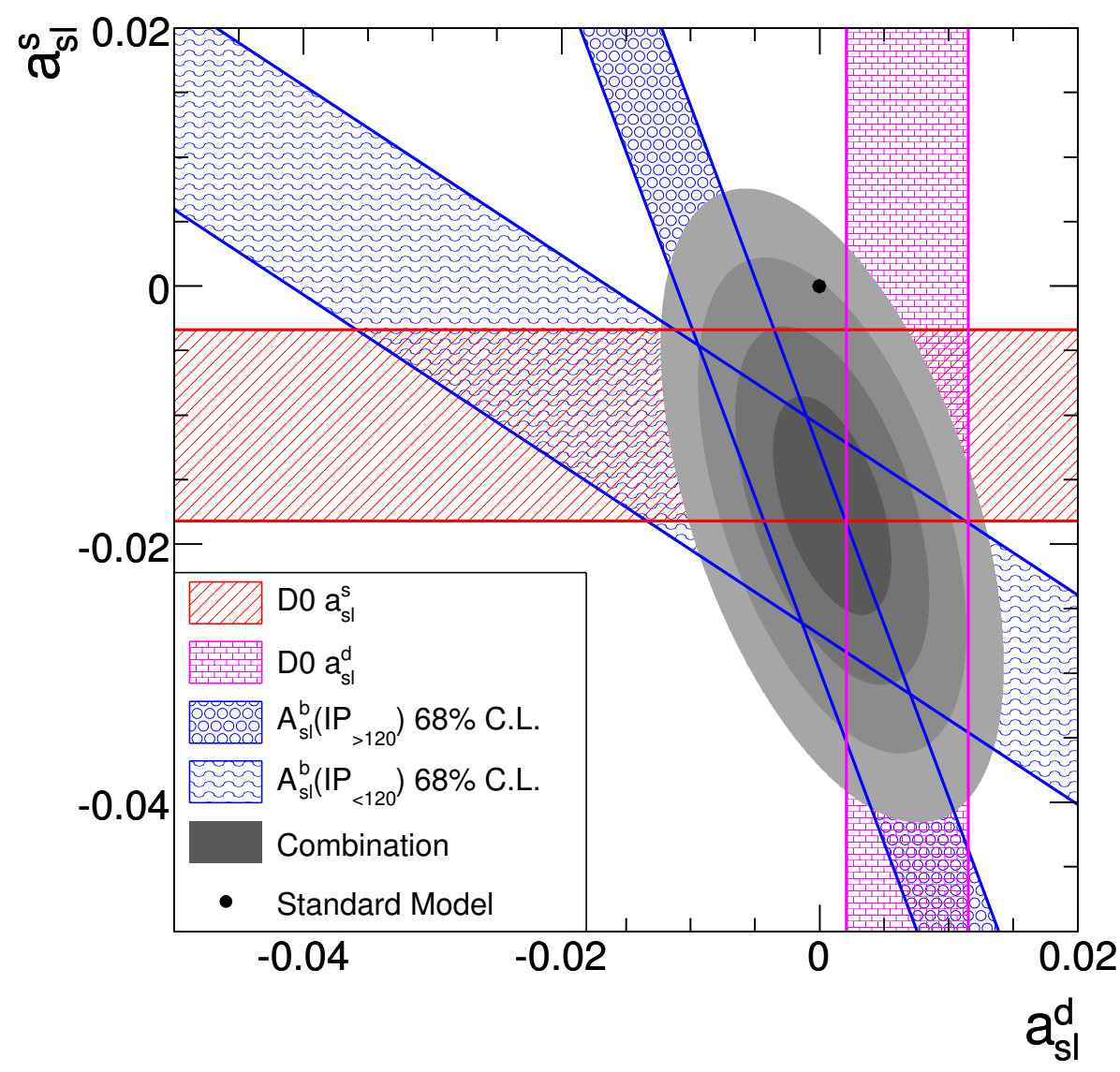

Figure 4: Combination of measurements of the D0 measurements of $a_{\mathrm{sl}}^{d}, a_{\mathrm{sl}}^{s}$, and the two impact-parameterbinned constraints from the same-charge dimuon asymmetry $A_{\mathrm{sl}}^{b}$ (D0 [3]). The bands represent the \pm 1 standard deviation uncertainties on each measurement. The ellipses represent the 1,2,3, and 4 standard deviation two-dimensional confidence level regions of the combination.

[3] V. M. Abazov et al. (D0 Collaboration), Phys. Rev. D 84, 052007 (2011).

[4] V. M. Abazov et al. (D0 Collaboration), Phys. Rev. Lett., 110, 011801 (2013).

[5] V. M. Abazov et al. (D0 Collaboration), Phys. Rev. D. 86, 072009 (2012).

[6] V.M. Abazov et al. (D0 Collaboration), Nucl. Instrum. Methods Phys. Res. A 565, 463 (2006).

[7] LHCb Collaboration, LHCb-CONF-2012-022 (2012, unpublished).

[8] C. Cartaro, CP and T violation at BaBar and Belle in the proceedings of 14th International Conference on B-Physics at Hadron Machines, POS (Beauty 2013) 006

[9] J. Beringer et al. (Particle Data Group), Phys. Rev. D 86, 010001 (2012). 


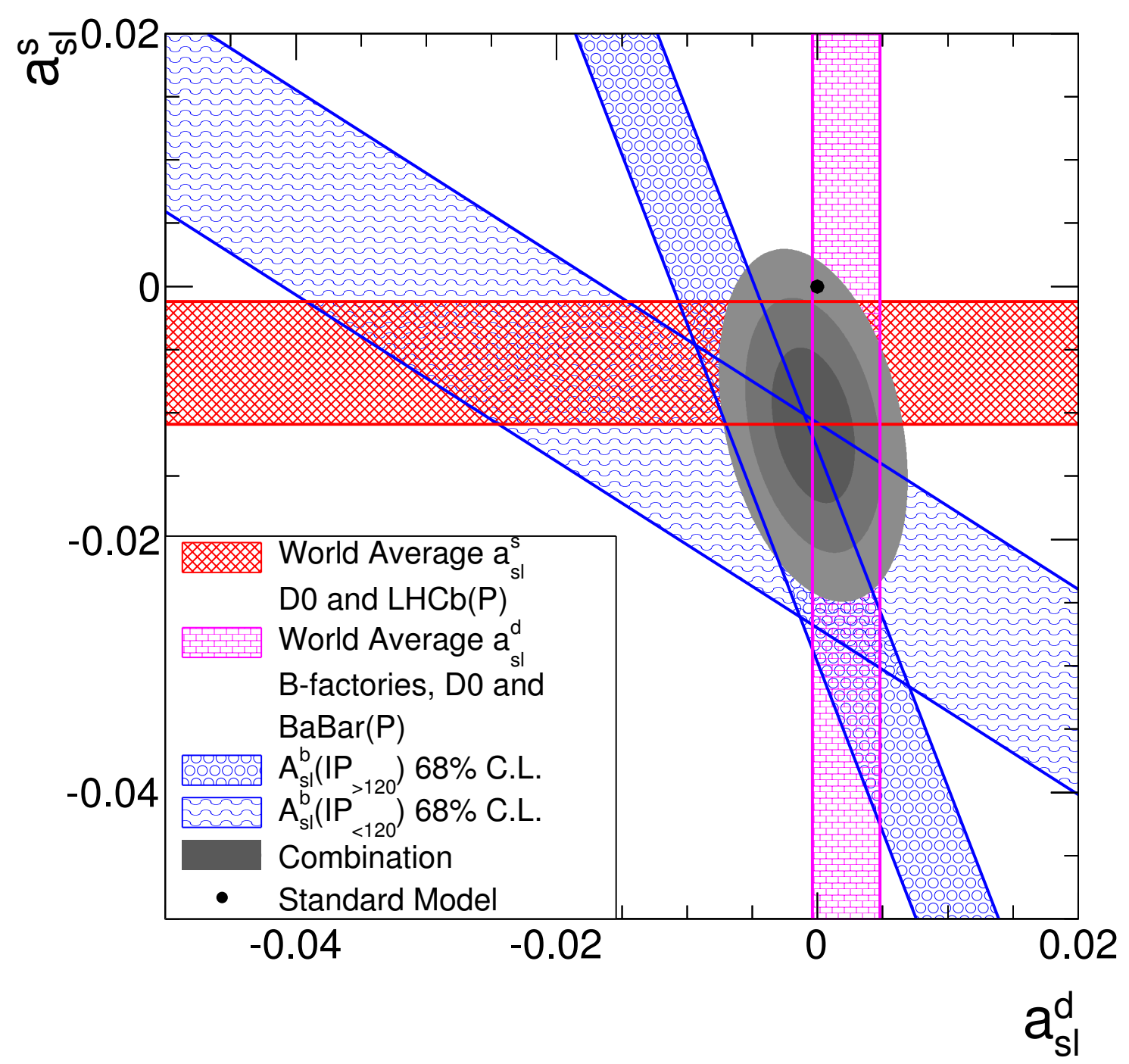

Figure 5: Combination of measurements of the new world averages of $a_{\mathrm{sl}}^{s}$ (D0 [4] and LHCb [7]), $a_{\mathrm{sl}}^{d}(B$ factories average [9], D0 [5] and the new BaBar result [8]), and the two impact-parameter-binned constraints from the same-charge dimuon asymmetry $A_{\mathrm{sl}}^{b}$ (D0 [3]). The bands represent the \pm 1 standard deviation uncertainties on each measurement. The ellipses represent the 1,2, and 3 standard deviation two-dimensional confidence level regions of the combination. 